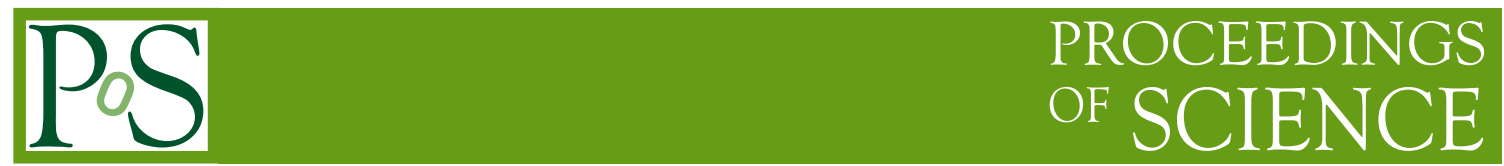

\title{
Current status of the space mission Millimetron
}

\author{
S. Pilipenko*, A. Smirnov, N. Kardashev
}

Lebedev Physical Institute, AstroSpace Center, Russia

E-mail: spilipenkodasc.rssi.ru

\begin{abstract}
Millimetron is a planned space observatory working in millimeter, submillimeter and far infrared bands with 10-m actively cooled mirror. It will operate in two modes. In the single dish mode with very high sensitivity Millimetron will address the key problems of cosmology, star and planet formation, interstellar medium and galaxy evolution left open by previous missions. The second mode is the Space-Earth radio interferometer with extremely high angular resolution and it is aimed at observations of the sub horizon scale structures of black holes.
\end{abstract}

Frontier Research in Astrophysics

26 - 31 May, 2014

Mondello (Palermo), Italy

* Speaker. 


\section{Introduction}

The interest of astronomers to millimeter, submillimeter, far infrared bands is explained by the following features:

- the CMB radiation maximum is located near the wavelength of $1 \mathrm{~mm}$;

- the maximum of radiation of coldest objects in the Universe, including dust clouds, asteroids, comets, is located in these bands;

- the minimum of the sky brightness is located near $300 \mu \mathrm{m}$, what allows to study very weak objects;

- these bands are very interesting for the atomic and molecular spectroscopy;

- the medium in these bands is more transparent than in the radio, near infrared and optical bands;

- the significant increase of the angular resolution of Very Long Base Interferometry (VLBI) can be achieved in millimeter and submillimeter bands.

However, the observations in these bands are very technically demanding since the Earth's atmosphere is opaque at wavelengths shorter than $300 \mu \mathrm{m}$. The recent progress in observations in these bands is connected with the Herschel space telescope (Pilbratt et al. 2003) and ALMA observatory (http://www.almaobservatory.org). The results of Herschel has brought a lot of new knowledge on the mechanism of star formation, properties of interstellar medium in our and other galaxies, atmospheres of bodies of the Solar System, etc. However, the limited angular resolution and sensitivity of Herschel with its $3.5 \mathrm{~m}$ main mirror cooled only to $70 \mathrm{~K}$ was insufficient for many important problems, see below in the Section 2. These problems also cannot be solved by ground-based facilities of the near future, e.g. ALMA which has very high angular resolution and sensitivity, but is limited by the Earth's atmosphere. Consequently, the next generation space mission, operating in submillimeter and far infrared is needed. Finally, recent advances in VLBI, namely the Radioastron Space-VLBI mission (Kardashev et al. 2013), the Event Horizon Telescope (http://www.eventhorizontelescope.org/) allow to expect that with the millimeter Space VLBI the horizon vicinity of black holes can be studied in great detail. These considerations lead to the concept of the Millimetron observatory, which is described in the Section 3. The summary of the current state of affairs in the development of Millimetron is presented in the Section 4.

\section{Scientific objectives}

Millimetron is designed as an all-purpose observatory which will be capable to meet the demands of astronomers in various fields. A review of suggestions of scientific proposals for Millimetron can be found in Kardashev et al. 2014 paper. Here we briefly discuss only the few science cases which are the most important in our view and unique for Millimetron.

The studies of the near Universe (the Solar System, our Galaxy and several nearby galaxies) with Millimetron will answer the questions of the distribution of the Hydrogen and water in the 
various objects, their dynamics and chemical composition. The studies of [CII] $158 \mu \mathrm{m}$ and HD 112 and $56 \mu \mathrm{m}$ spectral lines produced by the Inter Stellar Medium (ISM) will reveal the distribution of the hidden warm Hydrogen, which cannot be detected by other means. HD detection is also considered as the best way to measure the masses of protoplanetary disks (Bergin et al. 2013). These studies require the observatory to be capable for the high resolution spectroscopy on frequencies up to $6 \mathrm{THz}$.

One of the impressive results of Herschel telescope was the discovery of the fact that all the star formation takes place in thin filaments (Arzoumanian et al. 2011). However, the origin of these filaments, and, in particular, the role of magnetic field in their formation is not clear. Observations of polarization of filament emission with Millimetron will shed light on this problem.

For the cosmology and the distant Universe, Millimetron will contribute significantly to the study of high redshift galaxies, filling the gap in the spectral coverage between ALMA and existing planned Near-to-Mid infrared telescopes: Spitzer, WISE, James Webb Space Telescope (http://www.jwst.nasa.gov/). In particular, with the help of the medium resolution grating spectrometer Millimetron will be the only observatory to study the most important [CII], [NII], [NIII], [OI], [OIII] spectral lines at redshifts $z \leq 5$ in the majority of galaxies which are too faint for ground-based telescopes. These lines probe the star formation rate and the properties of the ISM of distant galaxies. At higher redshifts Millimetron will search for $\mathrm{HD}$ and $\mathrm{H}_{2}$ rotational transition emission lines, which will give invaluable information on the properties of Population III stars and metal-poor galaxies. Such observations require very high sensitivity, of the order of $10^{-22} \mathrm{~W} / \mathrm{m}^{2}$.

Deep far infrared images will allow to find many new interesting targets for the subsequent spectroscopy by Millimetron and for the study in other bands, e.g. by extremely large optical telescopes. Far infrared colors can be effectively used to select high redshift galaxies (Dowell et al. 2014).

The high sensitivity of the spectrometry at $C M B$ frequencies, $0.5-3 \mathrm{~mm}$, will give unique information about the Suynyaev-Zeldovich effect and for the first time will allow the high precision decomposition of thermal and kinetic contributions to the effect for a large sample of galaxy clusters (thermal effect for $>10^{5}$ clusters, de Bernardis et al. 2012). This studies will allow to test the cosmological model and the fundamental predictions of physics and astrophysics (Colafrancesco et al. 2014a,b,c). Millimetron has the same aperture as Earth-based South Pole Telescope, but the cold mirror, absence of atmosphere and continuous spectral coverage will allow to derive the parameters of plasmas in galaxy clusters with at least an order of magnitude better precision (de Bernardis et al. 2012).

The black holes will be the main target for the interferometer mode, in which Millimetron will operate together with the Earth-based telescopes (ALMA and other telescopes which participate in the EHT project). A black hole itself is radiating nothing, but the adjacent material which is being accreted or accelerated is a source of radiation which is then lensed by a black hole. This lensing creates the so called shadow. Measuring its size and shape is the straightforward way to confirm the existence of black holes (as objects predicted by the General Relativity). The shadows of known black holes are predicted to be extremely small: $<40 \mu$ as, that's why the very high angular resolution is needed. For the Earth-based EHT only two sources, Sgr A* and M87 will be accessible. Millimetron due to its higher resolution should be able to detect shadow structures of at least ten black holes with different masses, accretion modes and viewing angles. 


\section{Space observatory concept}

\subsection{Telescope parameters and design}

In order to achieve the high sensitivity and angular resolution which are required by mentioned scientific objectives which was indicated in Sec.2, the telescope mirrors should be big and cooled to temperature about several Kelvins. The big mirror means that it should have the greatest possible aperture, but be however light and compact to be carried by a launch vehicle into orbit. The latter requirement can only be met by a telescope with a deployable primary mirror. The cooling of the telescope to several Kelvins allows to reach sensitivity limited by the natural sky background. The main parameters of the observatory are given in Table 1 . The concept of the observatory is shown in Figure 1.

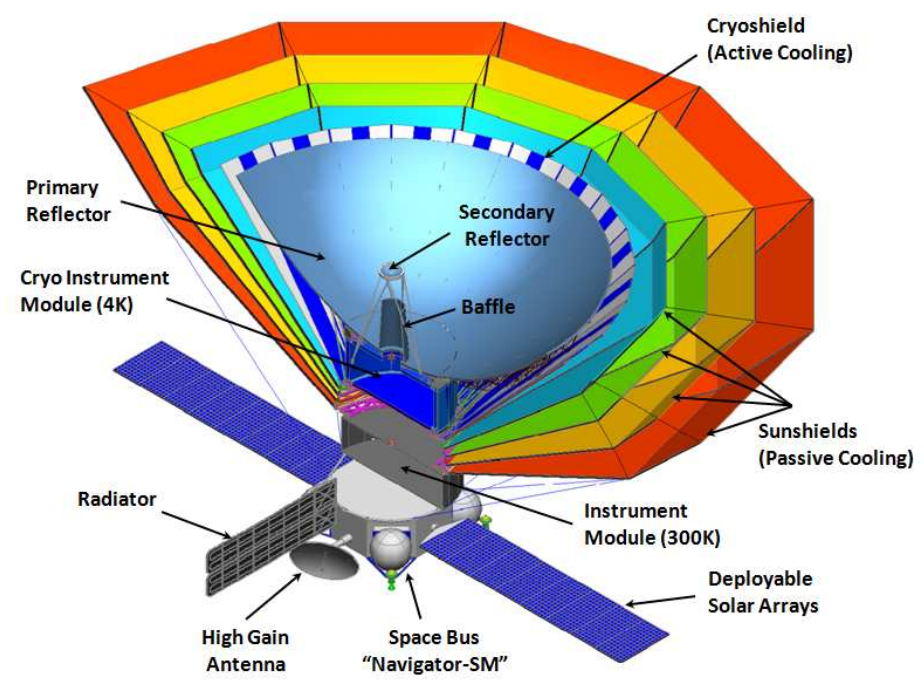

Figure 1: The conceptual design of the Millimetron.

The large aperture telescope is crucial for Millimetron, since it not only allows to increase the resolution (as $1 / D)$, but also to reduce the confusion limit created by the distant submillimeter galaxies which constitute the Cosmic Infrared Background (CIB). Millimetron will resolve $>90 \%$ of the CIB into individual sources at wavelengths up to $200 \mu \mathrm{m}$ (Smirnov et al. 2012).

The optical design of the telescope is a classical two-mirror Cassegrain system. Between the secondary mirror and focal plane is a third switching mirror. This mirror can be precisely and discretely rotated relative to the optical axis of the telescope and positioned to connect to various instruments. Cold space telescope requires minimization of the exterior thermal radiation. This justifies the short focus telescope design with the secondary mirror shielded by the deep primary mirror. The reflective surface of the primary mirror is formed by a central $3 \mathrm{~m}$ solid dish, and 24 outer deployable petals unfolded on-orbit. Each Millimetron's petals consist of three independent segments.Currently, the material of choice for the primary mirror is a high modulus carbon fibre, and a low temperature cyanate ester is supposed to be used as a binder.

For the Millimetron it is very important to achieve a high surface accuracy of the primary mirror after the deployment of the telescope in space. The deployment strategy is based on the 
previous successfully launched mission - RadioAstron, which has the same size and optimized for working in centimetre wavelengths. To achieve the required surface accuracy of the space telescope in the FIR, an active surface control system will be used to control all segments of the primary mirror with help of the wave-front sensing technique. This system will be periodically employed to correct inaccuracies in the positions of the deployed segments and variations of the overall surface caused by different factors.

The Millimetron spacecraft can be divided into two parts: the payload module and the bus module. We plan to use upgraded space bus module "Navigator" produced by the Lavochkin Association, Russia. Typically the "Navigator" includes service systems necessary for the spacecraft operation: on-board control system, a telecommunication system, power and propulsion systems. The bus module "Navigator" was designed to be adaptable to perform various missions and has been successfully used in several space mission. The Millimetron requires serious improvements of the space bus module "Navigator". The main of them concern a substantial improvement of pointing accuracy and stability of the guidance system, a power supply system will be changed on to the new energetic module to increased overall power consumption up to $6 \mathrm{~kW}$ and a propulsion system will be changed from the monocomponent to the two component liquid propulsion system.

Launch services will be provided by Russia (Russian Space Agency) since the Millimetron mission is included in the Russian Federal Space Program. The total mass of the spacecraft is about 6.6 tons and launch vehicle Proton in combination with a booster, are fully satisfactory to carry the spacecraft into orbit.

As we indicated above, cooling is one of the critical issues for the Millimetron mission. To achieve the required sensitivity of the telescope in the FIR we need to cool the antenna and the focal plane instruments down to about $4.5 \mathrm{~K}$. It may be possible to do this on-orbit only by a combination of effective radiation cooling and mechanical cooling. The telescope will be cooled passively to a temperature about $30-60 \mathrm{~K}$ by a suite of the deployable multi-layer heat shields. The closest to the

Table 1: Specifications of the Millimetron mission.

\begin{tabular}{|l|l|}
\hline Telescope aperture size & $10 \mathrm{~m}$ \\
\hline Telescope surface accuracy (RMS) & $\leq 10 \mu \mathrm{m}(\leq 5 \mu \mathrm{m}$ for the central solid dish) \\
\hline Telescope temperature & $\approx 4.5 \mathrm{~K}$ \\
\hline Wavelength range & $20 \mu \mathrm{m}-20 \mathrm{~mm}$ \\
\hline Cooling & effective radiation cooling + mechanical coolers \\
\hline Mode of observation & single-dish telescope or element SVLBI system \\
\hline Total mass & $\lesssim 6.6 \mathrm{t}$ \\
\hline Orbit & around Sun-Earth L2 Halo \\
\hline Life time & 10 years (aimed at 3-5 years with active cooling) \\
\hline Launch vehicle & Proton \\
\hline Launch year & 2020 \\
\hline
\end{tabular}


primary mirror cryogenic shield should be cooled to about $20 \mathrm{~K}$ by using $20-\mathrm{K}$ class mechanical coolers. That step reduce the radiation loads going from previous heat shields to primary mirror which will have the lower temperature. The 4-K class mechanical coolers will be used to cool the antenna as well as the focal plane instruments. Typically, that coolers consist of two-stage Stirling coolers and Joule-Thomson coolers with He-4 as operating gas. Sensitive detectors for FIR which will be placed in the instruments of the Millimetron require further deep cooling to temperatures about $50-100 \mathrm{mK}$. These temperatures will be generated within the instrument (as in the Herschel PACS scheme). May be it requires to use a 1-K class cooler, which consists of two-stage Stirling cooler and Joule-Thomson cooler with He-3 as operating gas. The mission life time with the active cooling system is estimated to be 3 - 5 years and depending of mechanical coolers lifetime.

\subsection{Scientific instruments}

The mission is going to cover a very wide wavelength range of the spectrum from the millimetre to the FIR with a four focal plane instruments: MHIFI (Millimetron Heterodyne Instrument for the Far Infrared), SACS (Short wave Array Camera/Spectrometer), LACS (Long wave Array Camera/Spectrometer) and heterodyne receivers for SVLBI. The cold parts of all receivers will be installed within a cryogenic container in the focal plane of the telescope.

The MHIFI is a successor of the Herschel HIFI instrument. It will cover higher frequencies, up to $\sim 5.4 \mathrm{THz}(50 \mu \mathrm{m})$. A new approach for that type of instruments is a multi-pixel design up to 7 pixels. The mixer technology for the frequencies $<1 \mathrm{THz}$ will be SIS (Superconductor-InsulatorSuperconductor) and HEB (Hot Electron Bolometer) for the higher frequencies. We predicted sensitivity of the instrument about 5 quantum limits. The low frequency bands of the MHIFI (557752 and $752-950 \mathrm{G} \mathrm{Hz}$ ) can be also used as SVLBI receivers. Up to day the many parts of the instrument has high technology readiness level (TRL).

The SACS will operate at wavelengths $20-450 \mu \mathrm{m}$. It will contain a grating spectrometer with medium spectral resolution $(R \sim 1000)$ and at the same time an imaging camera with up to several thousand pixels and field of view $6 x 6$ arcmin at $>2 \mathrm{THz}$. The concept of spectrometer will be analogous to the BLISS instrument, which was proposed by JPL(USA) for SPICA mission (Bradford et al. 2006). This instrument allows to observe wide spectral range simultaneously with very high sensitivity reaching $10^{-22} \mathrm{~W} / \mathrm{m}^{2}(5 \sigma, 1$ hour integration). The required detector sensitivity in terms of Noise Equivalent Power (NEP) should be about $1 \times 10^{-19} \mathrm{~W} / \sqrt{\mathrm{Hz}}$ or less. As we mentioned above to obtain this sensitivity the detectors require deep cooling to temperatures of about $50-100 \mathrm{mK}$.

The LACS consists of a differential Fourier spectrometer (FTS) covering wavelengths from 0.3 to $3 \mathrm{~mm}$ and several bands imaging camera with number of pixels in the range from 6 to 100 . Since LACS works in the wavelength range where the CMB radiation (signal) plays dominated role, the requirements for sensitivity of the detectors for him can be relaxed to $10^{-18}-3 \times 10^{-17}$ $\mathrm{W} / \sqrt{H z}$.

Such type of instruments, FTSs, are optimized for measurements of faint brightness gradients at submm/millimeter wavelengths, and it is required to be imaging (feeding a detectors array in its focal plane), to have a wide frequency coverage, and to be differential, i.e. to measure the difference in brightness between the cluster direction and the surrounding directions, rejecting the bright isotropic background from the CMB. 
The disadvantage of the FTSs is that all the frequencies from the whole frequency range to be measured contribute to form the radiative background on the detector. As a result, radiation noise is higher than in the case of dispersion spectrometers, where spectral bins are focused on the detector one at a time, sequentially. In order to diminish this disadvantage, the background is reduced by splitting the frequency coverage in a few sub-bands, using dichroic iñAlters, and using one array for each sub-band.

The heterodyne receivers for SVLBI mode should cover bands of $1.35 \mathrm{~cm}(22 \mathrm{GHz})$ and ALMA bands 1 (33-50 GHz), 3 (84-119 GHz), 6 (211-275 GHz), and probably $9(602-720 \mathrm{GHz})$ and $10(787-950 \mathrm{GHz})$. The last two bands will be the parts of the MHIFI instrument, while the other bands will be separate instruments. For this mode of observation we plan to use an on-board maser reference (hydrogen maser) and an on-board formatter with memory up to 10TB. After onboard preliminary processing the high-speed down-link transmits the data to the ground for the further processing. All the receivers are sensitive to polarisation. The instantaneous bandwidth of all SVLBI receivers will be about $4 \mathrm{GHz}$ per polarization. The limitation of this bandwidth today goes from the on-board memory volume and and low data rates in communication channel.

\section{Discussion and conclusions}

Currently Millimetron is supported and approved by the Russian Space Agency. The telescope construction, mechanical design, the space bus module, the mirror technology, SVLBI receivers, sun shields and many other parts are now in the phase of development, verification and testing.

Acknowledgments This work is partially supported by Russian President grant for the scientific schools NSh-4235.2014.2 and the Russian Academy of Scineces program P-21.

\section{References}

[1] Arzoumanian D. et al. $A \& A \mathbf{5 2 9}$ (2011) 6

[2] Bergin E.A. et al. Nature 493 (2013) 644

[3] de Bernardis P., Colafrancesco S. A\&A 538 (538) 86

[4] Bradford C.M., Nakagawa T., the BLISS-SPICA Team New Astronomy Reviews 50 (2006) 221

[5] Colafrancesco S. et al. A\&A 566 (2014a) 42

[6] Colafrancesco S. \& Marchegiani P. A\&A 562 (2014b) 2

[7] Colafrancesco S. AAS 22334005 (2014c)

[8] Dowell, C. D., et al. ApJ 780 (2014) 75

[9] Kardashev N.S., Khartov V.V., Abramov V.V. et al. ARep 57 (2013) 153

[10] Kardashev N.S., Novikov I.D., Lukash V.N. et al. Physics Uspekhi 57 (2014) 12

[11] Pilbratt G.L. Society of Photo-Optical Instrumentation Engineers (SPIE) Conference Series ed. Mather J. C. 4850 (2003) 586

[12] Smirnov A.V., Baryshev A.M., Pilipenko S.V., Myshonkova N.V. et al. Society of Photo-Optical Instrumentation Engineers (SPIE) Conference Series eds. M.C. Clampin et al. 8442 (2012) 4 